\title{
How Dirac's Seminal Contributions Pave the Way for Comprehending Nature's Deeper Designs
}

\author{
Mani L. Bhaumik
}

Department of Physics, University of California, Los Angeles. E-mail:bhaumik@physics.ucla.edu

Editors: Zvi Bern \& Danko Georgiev

Article history: Submitted on October 27, 2019; Accepted on December 6, 2019; Published on December 17, 2019.

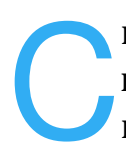

redible reasons are presented to reveal that many of the lingering century old enigmas, surrounding the behavior of at least an individual quantum particle, can be comprehended in terms of an objectively real specific wave function. This wave function is gleaned from the single particle energymomentum eigenstate offered by the theory of space filling universal quantum fields that is an inevitable outcome of Dirac's pioneering masterpiece. Examples of these well-known enigmas are wave particle duality, the de Broglie hypothesis, the uncertainty principle, wave function collapse, and predictions of measurement outcomes in terms of probability instead of certainty. Paul Dirac successfully incorporated special theory of relativity into quantum mechanics for the first time. This was accomplished through his ingenious use of matrices that allowed the equations of motion to maintain the necessary first order time derivative feature necessary for positive probability density. The ensuing Dirac equation for the electron led to the recognition of the mystifying quantized spin and magnetic moment as intrinsic properties in contrast to earlier ad hoc assumptions. The solution of his relativistic equation for the hydrogen atom produced results in perfect agreement with experimental data available at the time. The most far reaching prediction of the celebrated Dirac equation was the totally unexpected existence of anti-particles, culminating in the eventual development of the quantum field theory of the Standard Model that reveals the deepest secrets of the universe known to date. Quanta 2019; 8: 88-100.

\section{Introduction}

Just after getting his Ph.D. in 1926, the same year in which physics started to advance at breakneck speed following the successful formulation of quantum physics to resolve the perplexities of the atomic domain, Paul Dirac entered the field of research as a freshly minted scientific prodigy. Within a mere couple of years, he fashioned an elegant equation, ever to be known as the iconic Dirac equation of the electron. In contrast to extensive earlier efforts, his ingenious use of a special type of non-commutative $4 \times 4$ matrices allowed the relativistic quantum mechanical equations of motion to maintain the sought after hallmark first order time derivative characteristic of the Schrödinger nonrelativistic wave equation.

(C) 1 This is an open access article distributed under the terms of the Creative Commons Attribution License CC-BY-3.0, which permits unrestricted use, distribution, and reproduction in any medium, provided the original author and source are credited. 
This was a brilliant scheme for successfully combining the special theory of relativity with quantum mechanics to explain the behavior of electrons more accurately, avoiding the various problems encountered in earlier investigations.

First we present an extended overview of the emergence of Dirac's seminal equation, which had a profound impact for a large variety of topics including, most importantly, that seeded the eventual development of the quantum field theory of the Standard Model of particle physics. We will then focus on how the principles of the quantum field theory can be utilized for successfully uncovering the century old enigmas surrounding quantum physics.

In order to fully appreciate Dirac's momentous contribution, it would be helpful to briefly portray the particularly confounding circumstances of the period following the epochal unveiling of the Schrödinger's quantum mechanical wave equation. As early as in 1887, while trying to find a precise standard of length for use in their intended detection of ether drift, Albert A. Michelson and Edward W. Morley [1] observed that the red hydrogen spectral line is actually a closely spaced double line. The accuracy of measurement was so impressive that Michelson received the 1907 physics Nobel Prize for precision metrology and spectroscopy - and not, as might be commonly assumed, for the celebrated null result for ether drift that eventually provided one of the underpinnings for Einstein's formulation of special relativity.

The remarkable effectiveness of incorporating the special theory of relativity by Arnold Sommerfeld into the old quantum theory, before Schrödinger, was already evident in providing a more accurate agreement with experimental results of the hydrogen spectrum. Hence it was natural to seek a relativistic quantum mechanical wave equation. In fact, in his own attempt to formulate the quantum mechanical wave equation, Schrödinger first constructed a relativistic equation. He did not, however, publish this formulation since it did not agree with experimental results.

\section{The Klein-Gordon Equation}

In 1926, a number of authors independently formulated a relativistic wave equation, although the priority in publication belongs to Oscar Klein [2]. It is known today as the Klein-Gordon equation.

The Klein-Gordon equation is derived using the relativistic mass-energy equation

$$
E^{2}=m_{0}^{2} c^{4}+p^{2} c^{2}
$$

and drawing on the quantum mechanical energy and mo- mentum operators,

$$
E=\imath \hbar \frac{\partial}{\partial t}, \quad p=-\imath \hbar \frac{\partial}{\partial x}
$$

ensuing in

$$
-\hbar^{2} \frac{\partial^{2}}{\partial t^{2}}=m_{0}^{2} c^{4}-\hbar^{2} c^{2} \frac{\partial^{2}}{\partial x^{2}} .
$$

For a wave function $\psi$, dividing equation (3) by $\hbar^{2} c^{2}$ and rearranging the terms, the Klein-Gordon equation becomes

$$
\frac{1}{c^{2}} \frac{\partial^{2}}{\partial t^{2}} \psi-\nabla^{2} \psi+\frac{m_{0}^{2} c^{2}}{\hbar^{2}} \psi=0
$$

The equation (4) can be written in a graceful form

$$
\left(\square+\mu^{2}\right) \psi=0
$$

where $\mu=\frac{m_{0} c}{\hbar}$ and $\square$ is the d'Alembert operator,

$$
\bullet=\frac{1}{c^{2}} \frac{\partial^{2}}{\partial t^{2}}-\nabla^{2} .
$$

Soon the equation became the subject of numerous papers and was considered by many to be the correct and natural generalization of the Schrödinger equation. However, although it had a mathematical and aesthetic appeal, before long the limitation of its range of applicability became clear. To begin with, just like Schrödinger's earlier unpublished work, the equation failed to reproduce Sommerfeld's formula for the hydrogen spectrum that was in exact agreement with experiments. Possibly one of the biggest disappointments with the Klein-Gordon equation was its failure to account for the spin of the electron, as was also true of the nonrelativistic Schrödinger equation. However, it has been revived for use in the quantum field theory of spin 0 particles such as the Higgs boson.

Even before the emergence of the brave new world of contemporary quantum physics, some experimental observations were very baffling and required the introduction of electron spin to make sense of them. These are exemplified by the Stern-Gerlach experiment, the anomalous Zeeman effect, and Wolfgang Pauli's need for two additional quantum numbers to complete his Pauli exclusion principle, which provides a natural explanation for the periodic table of atoms.

In 1922, Otto Stern and Walther Gerlach conducted a rather remarkable experiment [3] to test the BohrSommerfeld model of the atom of the old quantum theory. A beam of silver atoms having a single electron in their outer shell was passed through a spatially varying magnetic field, which deflected them before they struck a detector screen. Stern and Gerlach found that the electrons were deflected discretely into only two clusters, a 
spectacular evidence of space quantization in an atom, supporting the evolving quantum paradigm at the time.

Niels Bohr was so startled that he personally wrote to Gerlach

I would be grateful if you or Stern could let me know, in a few lines, whether you interpret your experimental results in this way that the atoms are oriented only parallel or opposed, but not normal to the field, as one could provide theoretical reasons for the latter assertion. [4]

Furthermore, in some experiments with atoms in a magnetic field, spectral lines were observed to split into four, six, or even more lines and some triplets showed wider spacing than expected. These deviations were labeled "anomalous Zeeman effect" and were completely incomprehensible to early investigators.

Meanwhile Pauli devised the Pauli Exclusion Principle in 1925. The principle states that no two electrons can share the same quantum state at the same time [5]. This means that no two electrons in a single atom can have the same $n, \ell, m$, and $s$ numbers. However, the fourth quantum number was unknown at the time and he had to introduce it as an arbitrary assumption.

To explain all these conundrums, two graduate students, George Uhlenbeck and Samuel Goudsmit boldly hypothesized [6] that each electron spins with an angular momentum of one half Planck constant and carries a magnetic moment of one Bohr magneton. Spin is a quantum property of electrons without a classical analogue and is a form of angular momentum. The magnitude of this angular momentum is invariable.

Consequently, spin is now considered to be an intrinsic property of the electron with quantum number $s$ having values of $s=+\frac{1}{2}$ and $-\frac{1}{2}$. Along with Lorentz, Pauli initially was adamantly against the concept of rotation of a presumed rigid electron that could lead to a violation of relativity at the periphery. Goudsmith was so disappointed that he requested his thesis advisor Paul Ehrenfest not to submit their paper on spin for publication. Serendipitously, by then Ehrenfest had already submitted the paper and remarked that his students were young enough to accept some stupidity.

Eventually, Pauli relented and accepted the notion of the electron spin, as it gave a persuasive explanation for his two assumed additional quantum numbers as well as for the anomalous Zeeman Effect. The Stern-Gerlach experiment was also correctly explained as a consequence of the two distinct values of the electron spin quantum number. The result of the Stern-Gerlach experiment has been of abiding interest since no other experiment demonstrates such a graphic evidence of the quantized spin.
Pauli also presented a modified Schrödinger equation for spin $\frac{1}{2}$ particles taking into account the interaction of the particle's spin magnetic moment with an external magnetic field caused by the orbital motion of the electron [7]. This was a somewhat ad hoc modification to the Schrödinger equation to explain the existence of a doublet of additional energy levels attributable to the presence of spin. The natural occurrence of the electron spin and its magnetic moment still remained shrouded in a great mystery and the scientific community continued to devote significant efforts to find a cogent explanation of the conundrum. The relativistic Klein-Gordon equation offered no help either.

Perhaps the major concern with the Klein-Gordon equation was that the probability density given by the equation was not positive-definite. Apparently Dirac was the first to realize the problem with the probability interpretation for equations with second-order time derivatives. Also, because special relativity requires treating time and space on equal footing, Dirac reasoned that the equation has to be first order not only in time derivatives, but also in spatial derivatives. Instinctively, he persevered on finding an equation with these features.

While Dirac was focusing his full attention on the development of a relativistic quantum equation of motion in first order, to his great surprise, he did not receive any encouragement from the illustrious pioneer Niels Bohr. As Dirac recalls

I remember once when I was in Copenhagen, that Bohr asked me what I was working on and I told him that I was trying to get a satisfactory relativistic theory of the electron, and Bohr said 'But Klein and Gordon have already done that!' That answer first rather disturbed me. Bohr seemed quite satisfied by Klein's solution, but I was not because of the negative probabilities that it led to. I just kept on with it, worrying about getting a theory which would have only positive probabilities. [8, p. 690]

\section{The Dirac Equation}

To derive the equation, as usual Dirac started with the relativistic mass-energy equation (1), which using the Pythagoras relation $p^{2}=p_{x}^{2}+p_{y}^{2}+p_{z}^{2}$ yields

$$
E^{2}=m_{0}^{2} c^{4}+\left(p_{x}^{2}+p_{y}^{2}+p_{z}^{2}\right) c^{2}
$$

Getting the equation (1) in first order appeared to be a daunting task to most scientists of the time. Dirac was also very aware that a number of other physicists were working hard to construct a satisfactory relativistic 
quantum theory of the electron. It is just that time when Dirac's coup came along. As the story goes according to George Gamow [9, p. 125], the intuition to achieve the correct equation flashed in Dirac's mind one evening while he was staring into the fireplace at St John's College, Cambridge.

With his superb insight, Dirac ingeniously realized that the seemingly irresolvable conflict between the demands of relativity and his keenly perceived need for a first order equation in time derivative could be realized if he could find some matrices $\alpha_{0}, \alpha_{1}, \alpha_{2}, \alpha_{3}$ that would satisfy the relation,

$$
\alpha_{0}^{2}=\alpha_{1}^{2}=\alpha_{2}^{2}=\alpha_{3}^{2}=1
$$

and the anti-commutation relations hold

$$
\alpha_{i} \alpha_{j}=-\alpha_{j} \alpha_{i}, \quad(i \neq j) .
$$

He could then construct an equation for energy

$$
E=\alpha_{0} m_{0} c^{2}+c\left(p_{x} \alpha_{1}+p_{y} \alpha_{2}+p_{z} \alpha_{3}\right)
$$

By taking the square of the equation $(10)$, one could get the relativistic energy equation (7). The matrices $\alpha_{0}, \alpha_{1}$, $\alpha_{2}, \alpha_{3}$ are $4 \times 4$ matrices as follows

$$
\begin{aligned}
\alpha_{0} & =\left(\begin{array}{cccc}
0 & 0 & 1 & 0 \\
0 & 0 & 0 & 1 \\
1 & 0 & 0 & 0 \\
0 & 1 & 0 & 0
\end{array}\right), \\
\alpha_{1} & =\left(\begin{array}{cccc}
0 & 1 & 0 & 0 \\
1 & 0 & 0 & 0 \\
0 & 0 & 0 & -1 \\
0 & 0 & -1 & 0
\end{array}\right), \\
\alpha_{2} & =\left(\begin{array}{cccc}
0 & -l & 0 & 0 \\
l & 0 & 0 & 0 \\
0 & 0 & 0 & \imath \\
0 & 0 & -l & 0
\end{array}\right), \\
\alpha_{3} & =\left(\begin{array}{cccc}
1 & 0 & 0 & 0 \\
0 & -1 & 0 & 0 \\
0 & 0 & -1 & 0 \\
0 & 0 & 0 & 1
\end{array}\right) .
\end{aligned}
$$

Dirac was unaware of the fact that this type of matrices had been developed by William K. Clifford in 1877 and even earlier in 1840 by William R. Hamilton to take square root of a second order wave operator, which was subsequently shown to be subtly relativistically invariant. To quote Sir Roger Penrose

It is perhaps not surprising that Dirac was unaware of Clifford's discoveries of over half a century earlier, because this work was not at all well known in 1920s, even to many specialists in algebra. Even if Dirac had known of Clifford's algebras before, this would not have dimmed the brilliance of the realization that such entities are of importance for the quantum mechanics of a spinning electron-this constituting a major and unexpected advance in physical understanding. [10, p. 619]

These matrices are appropriate to be introduced in quantum mechanics since they are like linear operators acting on the wave function similar to the actions of the noncommuting position and momentum operators. Rather unexpectedly, these special types of matrices must refer to and act upon some new degrees of freedom in addition to the usual position and momentum variables of the quantum particle. The new degrees of freedom describe the physical spin of the electron and were later found to be true for all the fermions of nature as well. In the Dirac equation, these matrices act as operators on the wave function $\psi$ dubbed spinor, a name apparently coined by Paul Ehrenfest, the thesis advisor of the fortunate duo Uhlenbeck and Goudsmit. What Dirac had efficaciously introduced in quantum mechanics was a powerful new formalism, known today as spinor calculus. The very name spinor invokes rotation. But how exactly the electron spins remains mysterious [11].

Again, using the energy and momentum operators (2), the Dirac equation for a wave function $\psi(x, y, z, t)$ becomes

$$
i \hbar \frac{\partial}{\partial t} \psi(x, y, z, t)=\left[m_{0} c^{2} \alpha_{0}-\imath \hbar c\left(\frac{\partial}{\partial x} \alpha_{1}+\frac{\partial}{\partial y} \alpha_{2}+\frac{\partial}{\partial z} \alpha_{3}\right)\right] \psi(x, y, z, t)
$$

This is the equation essentially in the form originally proposed by Dirac [12, p. 255]. It is rather helpful for an intuitive understanding of the Dirac equation. Equation (15) can be presented very elegantly by drawing on the momentum four-vector with its magnitude remaining in- variant under Lorentz transformation. As usual, we start with the relativistic energy-momentum relation (1), which after rearranging terms becomes

$$
\frac{E^{2}}{c^{2}}-p^{2}=m_{0}^{2} c^{2}
$$


or

$$
\left(\frac{E}{c}\right)^{2}-p_{x}^{2}-p_{y}^{2}-p_{z}^{2}=m_{0}^{2} c^{2} .
$$

Equation (17) represents the equation for 4-momentum, whose magnitude remains invariant under Lorenz transformation. By using Dirac $\gamma$ matrices,

$$
\begin{aligned}
\gamma_{0} & =\left(\begin{array}{cccc}
1 & 0 & 0 & 0 \\
0 & 1 & 0 & 0 \\
0 & 0 & -1 & 0 \\
0 & 0 & 0 & -1
\end{array}\right), \\
\gamma_{1} & =\left(\begin{array}{cccc}
0 & 0 & 0 & 1 \\
0 & 0 & 1 & 0 \\
0 & -1 & 0 & 0 \\
-1 & 0 & 0 & 0
\end{array}\right), \\
\gamma_{2} & =\left(\begin{array}{cccc}
0 & 0 & 0 & -\imath \\
0 & 0 & \imath & 0 \\
0 & \imath & 0 & 0 \\
-l & 0 & 0 & 0
\end{array}\right), \\
\gamma_{3} & =\left(\begin{array}{cccc}
0 & 0 & 1 & 0 \\
0 & 0 & 0 & -1 \\
-1 & 0 & 0 & 0 \\
0 & 1 & 0 & 0
\end{array}\right) .
\end{aligned}
$$

where

$$
\begin{gathered}
\gamma_{0}^{2}=1, \quad \gamma_{1}^{2}=\gamma_{2}^{2}=\gamma_{3}^{2}=-1, \\
\gamma_{i} \gamma_{j}=-\gamma_{j} \gamma_{i} \quad(i \neq j)
\end{gathered}
$$

we construct an equation

$$
\gamma_{0} \frac{E}{c}+\gamma_{1} p_{x}+\gamma_{2} p_{y}+\gamma_{3} p_{z}=m_{0} c
$$

where by squaring equation (24), we can get equation (17).

Again, using the energy and momentum operators (2), equation 24 becomes

$$
\imath \hbar\left(\gamma_{0} \frac{1}{c} \frac{\partial}{\partial t}-\gamma_{1} \frac{\partial}{\partial x}-\gamma_{2} \frac{\partial}{\partial y}-\gamma_{3} \frac{\partial}{\partial z}\right)=m_{0} c
$$

which in vector multiplication notation can be written as

$$
\imath \hbar\left(\gamma_{0} \frac{1}{c} \frac{\partial}{\partial t}-\gamma \cdot \nabla\right)=m_{0} c
$$

The quantity within parenthesis in equation 26 is called the Dirac operator that can be written as $\gamma^{\mu} \partial_{\mu}$ where $\gamma^{\mu}$ are Dirac matrices and $\partial_{\mu}$ is the relativistically invariant 4-gradient,

$$
\partial_{\mu}=\left(\frac{1}{c} \frac{\partial}{\partial t}, \nabla\right)
$$

Then equation (26) can be written elegantly as

$$
i \hbar \gamma^{\mu} \partial_{\mu}=m_{0} c
$$

With the Dirac operator operating on a wave function $\psi$, we get an eigenvalue equation

$$
\imath \hbar \gamma^{\mu} \partial_{\mu} \psi-m_{0} c \psi=0
$$

Using natural units $\hbar=c=1$ and the Feynman slash notation $\not=\gamma^{\mu} \partial_{\mu}$, the equation becomes

$$
\left(\iota \not \supset-m_{0}\right) \psi=0
$$

This is one of the most graceful equations, which is just about as well known for its stunningly magnificent beauty as for its dramatic prediction for the deeper secrets of nature. Its physical consequences are more extensive and far-reaching than anyone could have imagined. A great deal more was hidden in the Dirac equation than even the author himself anticipated.

\section{Solution of Dirac Equation}

Since the Hamiltonian is a $4 \times 4$ matrix, the wave-function $\psi(r, t)$ it acts on is naturally a 4-component column vector:

$$
\psi(r, t)=\left(\begin{array}{l}
\psi_{1}(r, t) \\
\psi_{2}(r, t) \\
\psi_{3}(r, t) \\
\psi_{4}(r, t)
\end{array}\right)
$$

which gives a set of four coupled linear equations. The four-component wave function represents a new class of mathematical object in physical theories and makes their first appearance in Dirac's masterpiece.

Shortly after its earliest presentation [13], Dirac equation for the electron was solved for the hydrogen atom by Gordon [14] and Darwin [15] and subsequently by others. Now the results appear in contemporary textbooks [16, 17]. A summary of the results is presented below:

(a) Dirac's initial goal of developing a quantum theory of the electron with positive definite probability in a relativistic quantum theory was achieved with a first order time derivative.

(b) As an immediate consequence of his equation, the paradox of the mysterious quantized spin and the associated magnetic moment of the electron were understood to be a natural outcome of the Dirac equation. In the rest frame, the spin operator $\hat{s}$ for the Dirac particle, having eigenvalues $\pm \frac{1}{2} \hbar$, necessarily has an intrinsic spin, $s=\frac{1}{2}$, that is not related to ordinary orbital angular momentum $\ell$. However, the spin operator does not commute with the Hamiltonian in any frame other than the rest frame, $p=0$, so the expectation value of the spin operator is not a conserved quantity for $p \neq 0$. This agrees with the Stern-Gerlach experiment, where the single electron 
in the outermost shell having $\ell=0, m=0$, still shows deflection of the beam into only two discrete values of its magnetic moment relating to $s= \pm \frac{1}{2}$ and confirming that spin is an intrinsic property of the electron that was enigmatic even to Pauli himself.

Similarly, the orbital angular momentum operator $\hat{L}$ does not commute with the Hamiltonian in any frame other than the rest frame, so orbital momentum is not a conserved quantity either. However, the operator $\hat{J}=\hat{L}+\hat{s}$ commutes with the Hamiltonian in all frames. This strongly suggests that $\hat{J}$ should be interpreted as the operator for the total angular momentum, which is conserved.

(c) The gyro magnetic ratio of the electron spin turned out to be very close to 2 . But its spin angular momentum $\frac{1}{2} \hbar$ compensates giving an effective value of $g=1$ as previously assumed.

(d) The solution of the problem of the central field, which can be carried out exactly, leads to the prediction of the fine details of the spectrum of the hydrogen atom in rigorous agreement with the experimental results.

(e) The two unexpected extra equations resulting from the four component wave function seemed very perplexing. Two equations were enough to explain the two values of the spin as in Pauli's earlier provisional theory.

What then the other two spin values signify? Furthermore, the two extra equations seem to represent negative energy states. Even classically the negative energy states are indicated when we take the square root of equation (11). They are usually discarded as non-physical. However, doing so in quantum mechanics could be risky since complex numbers are used. But accepting them would make transitions from positive to negative energy states possible. Seemingly out of desperation, Dirac proposed his uncanny sea of electrons. Presumably all the possible states of the electron in the universe would be filled following Pauli's exclusion principle. One missing electron from the sea would be a hole into which an electron could transition. In spite of its obvious criticism, this strange idea persisted for a rather long time until Julian Schwinger proposed to retire it as an historical oddity.

Dirac then toyed with the idea that the two negative energy states could be for protons, which were the only positively charged particles known at the time. Evidently that idea also ran into trouble since the protons are about two thousand times heavier than the electrons. Dirac finally hit upon an unexpected winner. Seemingly the very baffling problem at its inception turned out to be a colossal discovery hidden in the equations. After the early confusion, a startling corollary of Dirac's equation yielded the prediction [18] of a new particle, the positron, an antimatter counterpart of the electron! The spins in the two additional equations belonged to two positrons.
The new particle would have the same mass as the electron but with opposite electric charge, and be capable of annihilating an electron, both being converted into pure energy in the process. Conversely, an electron and positron pair could be created provided there was enough energy available, unveiling a totally new vista of nature's mysteries. Exactly such an occurrence was soon discovered by Carl Anderson in 1932 from a meticulous analysis of cosmic ray tracks in a cloud chamber [19].

The physics Laureate Steven Weinberg affirms

The discovery of the more-or-less predicted positrons, together with the earlier success of the Dirac equation in accounting for the magnetic moment of the electron and the fine structure of hydrogen, gave Dirac's theory a prestige that it has held for over six decades. [20, p. 13]

\section{And Franck Wilczek articulates}

Dirac searched for a mathematical equation satisfying physically motivated hypotheses. $\mathrm{He}$ found that to do so he actually needed a system of equations, with four components. This was a surprise. Two components were most welcome, as they clearly represented the two possible directions of an electron's spin. But the extra doubling at first had no convincing physical interpretation. Indeed, it undermined the assumed meaning of the equation. Yet the equation had taken on a life of its own, transcending the ideas that gave birth to it, and before very long the two extra components were recognized to portend the spinning positron, as we saw.

With this convergence, I think, we reach the heart of Dirac's method in reaching the Dirac equation, which was likewise Maxwell's in reaching the Maxwell equations, and Einstein's in reaching both the special and the general theories of relativity. [21, p. 185]

Is it then any wonder that Dirac's accomplishment has been described as fully on a par with the works of Newton, Maxwell, and Einstein before him [22, p. 228].

\section{Harbinger of Quantum Field Theory}

While there are many aspects of Dirac's equation that led to uncovering the riddles of nature, we will now focus on how his seminal work initiated the development of the Quantum Field Theory that steers us to the deeper mysteries of nature revealed to date. 
The groundbreaking discovery of creation and annihilation of particle-antiparticle pairs indeed led to a radical change in our perception of nature, guiding us to the conclusion that particles could not be the primary reality of the universe as had been intuitively presumed so far. What then is the primary reality? Again, Dirac's pioneering contribution eventually guided us to the answer. As Anthony Zee articulates

It is in the peculiar confluence of special relativity and quantum mechanics that a new set of phenomena arises: Particles can be born and particles can die. It is this matter of birth, life, and death that requires the development of a new subject in physics, that of quantum field theory. [23, p. 4]

The very foundation of our understanding of the deeper secrets of nature is thus provided by successfully combining relativity with quantum mechanics in a way that even Dirac did not foresee when he embarked on the project.

Since the properties of a particle like an electron are exactly the same throughout the universe, a natural inference was that an underlying space filling quantum field is the primary reality, a quantized excitation of which appears as a particle. Thus Dirac's pivotal discovery eventually led to the new paradigm of Quantum Field Theory (QFT) that presents a unified view of nature, obliterating our long held perception that matter particles and forces possess totally different attributes.

Additionally, it was Dirac's innovative work leading to the first successful quantization of the electromagnetic field [24] that also gave a thoroughly quantummechanical treatment of spontaneous emission. Furthermore, Dirac also coined the name Quantum Electrodynamics for the simplest quantum field theory. Unfortunately, the theory got hopelessly bogged down for quite some time due to unexpected difficulties arising from the lack of cancellation of infinities.

Motivated by some key new experimental observations, particularly those of Willis Lamb [25] and P. Kusch [26], a new generation of young scientists predominantly Julian Schwinger [27], Richard Feynman [28], Shinichiro Tomonaga [29] and Freeman Dyson [30] succeeded in eliminating the infinities by utilizing the technique of renormalization. Following this achievement, it was natural to make attempts to develop a Quantum Field Theory that would encompass not only photons, electrons and positrons but also the plethora of other particles that were continually being discovered at the time in particle accelerators and cosmic rays together with the weak and strong nuclear forces that act upon them.

With contributions from many outstanding physicists, some of whom garnered Nobel Prizes for their excep- tional efforts throughout the second half of the twentieth century, we now have such a quantum field theory, known as the Standard Model of particle physics, which is a comprehensively expanded version of the quantum electrodynamics initiated by Paul Dirac. Experimentally verifiable accounts of the deepest design of nature revealed to date rest upon this quantum field theory of Standard Model. Needless to say, it by no means provides a complete account. Most conspicuously, it does not include gravity nor does it account for dark matter, dark energy, and possibly others that we have not glimpsed yet. However, Steven Weinberg affirms

We can be certain that the Standard Model will appear as at least an approximate feature of any better future theory. [31, p. 264]

Based on the discoveries completed to date, we now make a plausible effort to understand the various enigmas of the quantum world. Quantum theory is the most successful and supremely accurate theory propounded in recorded history. Yet, famously, certain aspects of the theory appear as weird and counter intuitive. Fortunately, I believe this "weirdness" can be understood from the precepts of QFT that portrays a reality different from our daily classical reality, both coexisting by transitioning from the quantum to the classical domain.

\section{The wave function of an electron}

According to QFT, a particle like an electron arises as a quantized excitation of the underlying electron quantum field. Such an energy-momentum eigenstate of the field can be expressed as a specific Lorentz covariant superposition of field shapes of the electron field along with all the other quantum fields of the Standard Model of particle physics. Superposition of field shapes in a one-particle state is not stationary in time but evolve in a simple wavelike manner.

The individual field shapes, each with their own computable dynamic time evolution, are actually the vacuum fluctuations comprising the very structure of the energy-momentum eigenstate. The vacuum fluctuations are evanescent in the sense that they pass away soon after coming into being. But new ones are constantly boiling up to establish an equilibrium distribution so stable that their contribution to the electron $g$-factor results in a measurement accuracy of one part in a trillion [32]. The Lorentz covariant superposition of vacuum fluctuations of all the quantum fields in the one-particle quantum state can therefore be conveniently represented using the Fourier synthesis approach leading to a well behaved smooth wave packet that is everywhere continuous and continuously differentiable. 
The wave function $\psi(x)$, for simplicity in one dimension, will be given by the Fourier integral

$$
\psi(x)=\frac{1}{\sqrt{2 \pi}} \int_{-\infty}^{\infty} \tilde{\psi}(k) e^{\imath k x} d k
$$

where $\tilde{\psi}(k)$ is a function that quantifies the amount of each wave number component $k=\frac{2 \pi}{\lambda}$ that gets added to the combination.

From Fourier analysis, we also know that the spatial wave function $\psi(x)$ and the wave number function $\tilde{\psi}(k)$ are a Fourier transform pair. Therefore, we can find the wave number function through the Fourier transform of $\psi(x)$ as

$$
\tilde{\psi}(k)=\frac{1}{\sqrt{2 \pi}} \int_{-\infty}^{\infty} \psi(x) e^{-l k x} d x
$$

Thus the Fourier transform relationship between $\psi(x)$ and $\tilde{\psi}(k)$, where $x$ and $k$ are known as conjugate variables, can help us determine the frequency or the wave number content of any spatial wave function. In order to determine the time evolution of the wave packet function, we need to incorporate the time term to the spatial function. Accordingly,

$$
\psi(x, t)=\frac{1}{\sqrt{2 \pi}} \int_{-\infty}^{\infty} \tilde{\psi}(k) e^{i(k x-\omega t)} d k
$$

where $\omega=2 \pi v$ is the angular frequency.

A wave packet or a wave function is localized and therefore can represent a quantum particle, but just holistically, since only the totality of the wave packet represents all the conserved quantities of the energy-momentum eigenstate of a particle such as mass, charge, and spin. Creation of a similar wave function of a particle has been presented earlier [33] from a rather exploratory view for a better intuitive understanding.

When quantum mechanics with its hallmark wave function supervened on the atomic domain of physics and commenced explaining its mysterious workings with uncanny consistency, it appeared totally contrary to our intuition developed from classical physics. Even a century later, quantum mechanics still perplexes most people including many scientists. However, there appear to be plausible answers to the riddles of quantum mechanics based on the discoveries of the quantum field theory of the Standard Model, again the crown jewel of physics that was forged through, commencing with Paul Dirac's uncanny insight.

Following is a consolidated account of resolutions of the perplexities of quantum mechanics, some of which has been presented in parts by the author in previous publications [34-36]. For simplicity, here we will be dealing solely with a single quantum particle, a multi particle extension of which is under preparation and will be presented later. Since the depiction of the single particle wave function presented here is based on a fully relativistic account, its reality should be extendable to multi particle systems. Although some emergent properties like quantum entanglement appear in a multi particle system, the fundamental quantum realism of a single particle wave function should shore up the reality of the multi particle wave function, which for expediency is presented in a rather abstract Hilbert space formalism.

\subsection{Reality of the Wave Function}

From the early days of quantum mechanics, physicists have argued about the reality of the wave function. It is surprising to find them still debating it, particularly in light of Erwin Schrödinger's demonstration that the mysteriously discrete Bohr orbitals of the hydrogen atom arise from standing wave patterns of the wave function of the electron. Even after almost a century, it is not uncommon for very notable contemporary scientists to pronounce the wave packet for a particle like an electron to be nothing but a fictitious mathematical construct. Does not it stretch credibility to imagine that fictitious waves can possibly make such unmistakably robust standing waves to form discrete orbitals of atoms?

It is also hard to fathom how anyone can doubt the reality of a wave associated with a particle after an unambiguous experimental demonstration of the electron diffraction pattern of the de Broglie matter wave. Although de Broglie himself failed to recognize the reality of the observed wave, he correctly surmised the origin of the wave to be a result of the relativistic effect of an unspecified internal frequency associated with the particle. Now we know that the internal frequency relates to the relativistic equation: $v=\frac{m_{0} c^{2}}{h}$ indicating that a matter particle is itself a wave packet as is unmistakably evident from QFT.

Historically, the de Broglie hypothesis that a matter particle behaves like a wave of length, $\lambda$, related to its momentum, $p$, through the Planck constant, $h$ to be $\lambda=\frac{h}{p}$ or equivalently $p=\hbar k$ helped Schrödinger to develop his wave equation that supercharged the brand new discipline of quantum mechanics. Using the Lorentz invariant property of the wave packet, the relation $p=\hbar k$ can indeed be derived irrespective of the mass of the particle [34] and therefore need not be a mere hypothesis.

As described earlier, a quantum particle is a holistic wave packet consisting of irregular disturbances of the various quantum fields of the Standard Model and these disturbances are not readily measurable. Their distinct consequences, however, are by now well established for explaining the Lamb shift and the anomalous magnetic moment of the electron. These disturbances of the quantum fields otherwise known as vacuum fluctuations are 
like the 'beables' eponymously coined by John Stuart Bell that corresponds to something that really exists physically but not directly observable. In Bell's words

The beables of the theory are those elements which might correspond to elements of reality, to things which exist. Their existence does not depend on 'observation'. [37, p. 174]

Quarks and gluons are distinct examples of beables. No one can see an isolated quark or a gluon, but few scientists doubt their real existence.

Some graphic depiction of the vacuum fluctuations could as well be offered soon. The 2014 Kavli Prize in Astrophysics was awarded for pioneering contributions to the theory of cosmic inflation. The predictions of the simplest versions of the theory have been so successful that most cosmologists accept that some form of inflation truly did occur in the very early universe. Assuming the veracity of cosmic inflation at the dawn of the universe, the imprint of the disturbances of the quantum field could manifestly be discernible as immensely enlarged vacuum fluctuations in the cosmic microwave background radiation anisotropy, recorded both by the WMAP and Planck satellite [38].

Since the quantum fields manifestly comprise a reality of the universe, their disturbances should be no less real. Thus the mere straightforward immeasurability of the disturbances of the quantum fields does not make them fictitious, providing distinct validation for the wave function portrayed here to be objectively real. There is every reason to believe that the very weave of our universe supports the objective reality of the depicted wave function, which represents a natural phenomenon and not just a mathematical postulate.

The perplexing wave-particle duality should by now be comprehensible. In reality, there is no such thing in the quantum domain as a classical particle akin to a tiny marble ball. What we call a quantum particle is actually a wave packet consisting of real field disturbances that in their totality behave as a particle. The idea of a particle, however, is so ingrained in our awareness that it is unlikely to fade away anytime soon. This is especially true since at energies higher than that corresponding to the Compton wavelength, the exchange of momentum is more particle-like, as in inelastic scattering. Also the visible tracks of the high energy elementary particles in a particle detector like a cloud chamber are particle-like.

\subsection{The Uncertainty Principle}

On the basis of those characteristics of the wave function that represent a quantum particle, the mysterious uncertainty principle of quantum physics can now be understood as a perfectly natural incidence. The Fourier transform correlations between conjugate variable pairs of any wave packet have powerful consequences since these variables obey the uncertainty relation

$$
\Delta x \Delta k \geq \frac{1}{2}
$$

where $\Delta x$ and $\Delta k$ relate to the standard deviations $\sigma_{x}$ and $\sigma_{k}$ of the wave packet. This is a completely general property of a wave packet with a reality of its own and is in fact inherent in the properties of all wave-like systems. It becomes important in quantum mechanics because of the real wave nature of particles having the relationship $p=\hbar k$, where $p$ is the momentum of the particle. Substituting this in the general uncertainty relationship of a wave packet, the intrinsic uncertainty relation in quantum mechanics becomes

$$
\Delta x \Delta p \geq \frac{1}{2} \hbar
$$

This uncertainty relationship has been conflated with a somewhat analogous observer effect, which advocates that measurement of certain systems cannot be made without affecting the system. In fact, Heisenberg offered such an observer effect in the quantum domain as a "physical explanation" of quantum uncertainty, an explanation that has since gone by the name of Heisenberg's uncertainty principle. What the uncertainty principle actually states, however, is a fundamental property of quantum systems, and is not a statement about observational indeterminacy as was suggested by Heisenberg, indeed originally branding it the indeterminacy principle. In fact, some recent studies [39-41] highlight important fundamental differences between uncertainties in quantum systems and the limitation of measurement in quantum mechanics.

Einstein's fundamental objection to the Copenhagen view of the wave packet was its assertion that any underlying reality of the uncertainties was irrelevant and should be accepted under the veil of complementarity. We have established that this uncertainty is indeed an essential reality, governed by wave behavior, traceable in its origin back to the wave-particle duality first envisioned and steadfastly maintained as a reality by Einstein, alone among his peers, for over a decade.

\subsection{Born's Rule of Probability}

Perhaps the most perplexing aspect of quantum physics is its probabilistic prediction, as formalized by Born's rule. From experiments on the scattering of electrons, Max Born showed that the original suggestion of Schrödinger that the wave packet be considered as the charge distribution of the electron could not be justified. Instead, Born 
followed Einstein in this regard as he stated in his Nobel lecture

Again an idea of Einstein's gave me the lead. He had tried to make the duality of particles (light quanta or photons) and waves comprehensible by interpreting the square of the optical wave amplitudes as probability density for the occurrence of photons. This concept could at once be carried over to the $\psi$-function: $|\psi|^{2}$ ought to represent the probability density for electrons (or other particles). [42]

This inference is in accord with the correct nature of the wave function, as presented in Section 6.1. As the amplitudes of the wave packet consist of disturbances of various diverse quantum fields, they could not simply represent a distribution of charge or any other classical property of the particle. Since the absolute square of the amplitude is non-negative, whereas the amplitude generally contains complex numbers, $|\psi|^{2}$ should represent the probability density of a quantum particle like an electron. This is why the amplitude of the wave function is commonly called the quantum probability amplitude. Since the total probability must be one, we have to normalize the wave function as

$$
\int_{-\infty}^{\infty}|\psi(x, t)|^{2} d x=1
$$

Born's rule is inseparably connected to the perplexing measurement problem. Although its exact mechanism is intensely debated, the most popular paradigm appears to be that of decoherence, first posited by H. Deter Zeh [43] and extensively studied by Wojciech Zurek [44] and others. In this model the quantum system to be measured gets entangled with the quantum constituents of the required macroscopic detector ultimately resulting in the selection of the measurable event.

Zurek contends [45] that the Born rule can actually be derived from the theory of decoherence as opposed to being a mere postulate of quantum theory. There is indeed some support for his contention [46] although why a particular probability out of many others materializes needs to be explained [47]. Quite possibly this is not definitively answerable because of the inherent, irreducible indeterminacy of the primordial quantum fluctuations that is irrefutably carried over to the elementary particles.

The baffling 'collapse of the wave function', however, has a genuine explanation based on the nature of the wave function described in Section 6.1. The holistic feature of the wave function requires that the entirety of the wave function be acquired all together or not at all. This is aided by the fact that the wave function of the particle is also entangled with space [48]. Hence, the spreadout wave function can instantaneously converge at the particular location of detection.

An example from cosmic history is worth examining in this regard. The universe about 380,000 years after the big bang consisted primarily of hydrogen ions (protons) and electrons, along with neutral helium atoms. An electron would naturally be attracted to the proton, starting to emit electromagnetic radiation due to its motion. But a much more rapid process would take place when the electron, while aligned in the direction of the proton, spontaneously emits a photon with an amount of energy that exactly matches the potential energy of the electron and an orbital of the hydrogen atom. In this process the wave function of the electron can directly wind up as the wave function of the particular orbital of the hydrogen atom without having to undergo a typical collapse to any particular point. Such episodes would reveal that the wave function does not necessarily always need to go through a traditional collapse for detection.

The above episode, which indeed took place in a universal scale, could be used as a pragmatic toy model for grasping the much discussed but yet to be resolved measurement problem. In this case, the necessary macroscopic detection device consists solely of essentially an assembly of the same quantum constituents, namely, atomic hydrogen ions. Also, the quantum state to be detected is a simple position wave function of the electron. Even without going into all the details, one can readily comprehend that in addition to predicting only the probability of detection instead of a certainty, there is also an inherent, irreducible element of indeterminacy in the occurrence of any particular probability since the predominant necessary event of the spontaneous emission of a photon of matching potential energy by the electron is totally unpredictable from the Born rule.

The counter-intuitive simultaneous presence of the electron in more than one place can also be understood in terms of the nature of the wave function. Since the wave function is spread out in space, which can be substantial due to dispersion, the probability of finding the particle in multiple places will be significant. Again, in each location the wave function has to be taken in its entirety, only that represents all the properties of the particle, thus giving an ostensible appearance of the electron in many places at the same time.

As a manifest evidence of the wave-particle duality, the famous double slit experiment, in which one quantum particle at a time is sent through two adjacent slits and results in a wave-like interference pattern, also has a natural explanation. The wave function consisting of linear superposition of amplitudes gets divided into two parts at the slits, which then interferes resulting in a typical wave 
diffraction pattern, yet detected only at a point with the characteristic totality of the wave packet. The amplitude of the wave packet can be split, but the square of the amplitude of the entire wave packet required for detection cannot be divided. Thus, each individual electron contributes one dot to an overall pattern that looks like the two-slit interference pattern of a wave. A distinctly typical interference pattern arises when a sufficient number of quantum particles are sent through the two slits either one at a time or all of them together at the same time.

\section{Conclusions}

We have expounded how Dirac's revolutionary equation has been invaluable in unveiling some of the bewildering mysteries of the atomic domain. A totally unexpected outcome of Dirac's equation of the electron led to the development of the quantum field theory of the Standard Model of particle physics, leading in turn to the deepest revelation to date, of nature's most profound secrets. We have shown that the ensuing quantum field theory originating from Dirac's pioneering contributions can assist in demystifying many of the lingering mysteries of quantum physics for both the professionals and the general public.

An objectively real wave function can be garnered from the edicts of the quantum field theory revealing that there are no solid elementary particles but only holistic wave packets acting like particles. Amazingly, the paradigm of the wave function presented here proffers plausible explanation for the long standing inherent perplexities of the wave-particle duality, uncertainty principle, de Broglie wave hypothesis, the bizarre wave function collapse, detecting probabilities instead of certainty.

These quantum oddities arise from our natural instinct to understand the quantum domain in terms of our daily classical reality. The quantum field theory reveals that the eventual quantum reality is substantially different from our familiar classical reality.

The quantum fields fill all space of the entire universe for all times. The fields have infinite degrees of freedom of creating and annihilating quantum particles locally at each spacetime element and these degrees of freedom are indeed always active obeying the energy-time uncertainty relation even in the complete vacuum. The quantum activities have the inherent special attribute that each event is totally spontaneous and completely unpredictable as to when it is going to take place. This is only a slow motion description of the events. In reality, there are infinite number of these evanescent events occurring locally at every spacetime element of the universe, the members of the ensemble having infinitely different amplitudes and speed.
This unique quantum activity, a feature of each one of the quantum fields of the Standard Model, being present everywhere, ubiquitously interact with everything in the quantum domain including elementary particles, atoms and molecules. The unique features such as the characteristic quantum superposition as well as some inherent unpredictability could be an inevitable result. Thus the reality of the quantum domain is substantially different.

However, the quantum domain transitions into our familiar daily macroscopic classical domain where nature deals with an innumerable number of particles that mask the quantum activity with phenomena like decoherence. Experiments confirm that the characteristic activities of the quantum domain are revealed when a fairly macroscopic object containing a trillion atoms is shielded from environmental decoherence as completely as possible. Thus the quantum reality is markedly different, but coexists by transitioning into classical reality. Much of the quantum enigmas disappear when we accept this fact of nature.

\section{Acknowledgment}

The author wishes to thank Per Kraus, Zvi Bern, Danko Georgiev, Eric D'Hoker and Joseph Rudnick for illuminating discussions.

\section{References}

[1] A. A. Michelson, E. W. Morley. On a method of making the wavelength of sodium light the actual and practical standard of length. American Journal of Science 1887; 34(204):427-430. doi : 10.2475/ ajs.s3-34.204.427.

[2] O. Klein. Quantentheorie und fünfdimensionale Relativitätstheorie. Zeitschrift für Physik 1926; 37(12):895-906. doi:10.1007/bf01397481.

[3] W. Gerlach, O. Stern. Das magnetische Moment des Silberatoms. Zeitschrift für Physik 1922; 9(1):353355. doi:10.1007/bf01326984.

[4] B. Friedrich, D. Herschbach. Stern and Gerlach: how a bad cigar helped reorient atomic physics. Physics Today 2003; 56(12):53-59. doi:10.1063/ 1.1650229 .

[5] W. Pauli. Über den Einfluß der Geschwindigkeitsabhängigkeit der Elektronenmasse auf den Zeemaneffekt. Zeitschrift für Physik 1925; 31(1):373-385. doi: $10.1007 / \mathrm{bf02980592.}$ 
[6] G. E. Uhlenbeck, S. Goudsmit. Ersetzung der Hypothese vom unmechanischen Zwang durch eine Forderung bezüglich des inneren Verhaltens jedes einzelnen Elektrons. Die Naturwissenschaften 1925; 13(47):953-954. doi : 10.1007/bf01558878.

[7] W. Pauli. Zur Quantenmechanik des magnetischen Elektrons. Zeitschrift für Physik 1927; 43(9):601623. doi : 10.1007/bf01397326.

[8] J. Mehra. The Golden Age of Theoretical Physics. Vol. 2. World Scientific, Singapore, 2001. doi: 10.1142/4454.

[9] G. Gamow. Thirty Years that Shook Physics: The Story of Quantum Theory. Doubleday and Co., Garden City, New York, 1966.

[10] R. Penrose. The Road to Reality: A Complete Guide to the Laws of the Universe. Jonathan Cape, London, 2004.

[11] C. T. Sebens. How electrons spin. Studies in History and Philosophy of Science Part B: Studies in History and Philosophy of Modern Physics 2019; 68:40-50. arXiv: 1806.01121, doi:10.1016/j. shpsb.2019.04.007.

[12] P. A. M. Dirac. The Principles of Quantum Mechanics. 4th Edition. Oxford University Press, Oxford, 1967.

[13] P. A. M. Dirac. The quantum theory of the electron. Proceedings of the Royal Society of London. Series A 1928; 117(778):610-624. doi:10.1098/rspa. 1928.0023 .

[14] W. Gordon. Die Energieniveaus des Wasserstoffatoms nach der Diracschen Quantentheorie des Elektrons. Zeitschrift für Physik 1928; 48(1):11-14. doi : 10.1007/bf01351570.

[15] C. G. Darwin. The wave equations of the electron. Proceedings of the Royal Society of London. Series A, Containing Papers of a Mathematical and Physical Character 1928; 118(780):654-680. doi:10.1098/rspa.1928.0076.

[16] B. Thaller. The Dirac Equation. Texts and Monographs in Physics. Springer, Berlin, 1992. doi: 10.1007/978-3-662-02753-0.

[17] V. G. Bagrov, D. Gitman. The Dirac Equation and its Solutions. De Gruyter Studies in Mathematical Physics. Walter de Gruyter, Berlin, 2014.
[18] P. A. M. Dirac. Quantised singularities in the electromagnetic field. Proceedings of the Royal Society of London. Series A, Containing Papers of a Mathematical and Physical Character 1931; 133(821):60-72. doi : 10.1098/rspa.1931.0130.

[19] C. D. Anderson. The positive electron. Physical Review 1933; 43(6):491-494. doi:10.1103/ PhysRev.43.491.

[20] S. Weinberg. The Quantum Theory of Fields. Vol. 1. Cambridge University Press, Cambridge, 2013. doi : 10.1017/cbo9781139644167.

[21] F. Wilczek. Fantastic Realities: 49 Mind Journeys and A Trip to Stockholm. World Scientific, Singapore, 2006. doi : 10.1142/6019.

[22] T. Hey, P. Walters. The New Quantum Universe. Cambridge University Press, Cambridge, 2013. doi: 10.1017/cbo9780511818752.

[23] A. Zee. Quantum Field Theory in a Nutshell. Princeton University Press, Princeton, New Jersey, 2003.

[24] P. A. M. Dirac. The quantum theory of the emission and absorption of radiation. Proceedings of the Royal Society of London. Series A, Containing Papers of a Mathematical and Physical Character 1927; 114(767):243-265. doi : 10. 1098/rspa. 1927.0039 .

[25] W. E. Lamb, R. C. Retherford. Fine structure of the hydrogen atom by a microwave method. Physical Review 1947; 72(3):241-243. doi : 10.1103/ PhysRev. 72.241

[26] P. Kusch, H. M. Foley. Precision measurement of the ratio of the atomic ' $g$ values' in the ${ }^{2} P_{\frac{3}{2}}$ and ${ }^{2} P_{\frac{1}{2}}$ states of gallium. Physical Review 1947; 72(12):1256-1257. doi:10.1103/PhysRev.72, 1256.2 .

[27] J. Schwinger. On quantum-electrodynamics and the magnetic moment of the electron. Physical Review 1948; 73(4):416-417. doi:10.1103/PhysRev. 73.416 .

[28] R. P. Feynman. A relativistic cut-off for classical electrodynamics. Physical Review 1948; 74(8):939946. doi : 10.1103/PhysRev. 74.939.

[29] S. Tomonaga. On a relativistically invariant formulation of the quantum theory of wave fields. Progress of Theoretical Physics 1946; 1(2):27-42. doi : 10.1143/ptp.1.27. 
[30] F. J. Dyson. The radiation theories of Tomonaga, Schwinger, and Feynman. Physical Review 1949; 75(3):486-502. doi:10.1103/PhysRev. 75.486.

[31] S. Weinberg. To Explain the World: The Discovery of Modern Science. HarperCollins, New York, 2015.

[32] D. Hanneke, S. Fogwell, G. Gabrielse. New measurement of the electron magnetic moment and the fine structure constant. Physical Review Letters 2008; 100(12):120801. doi:10.1103/ PhysRevLett. 100.120801

[33] M. L. Bhaumik. Was Albert Einstein wrong on quantum physics?. Quanta 2015; 4(1):35-42. doi : 10.12743/quanta.v4i1.47

[34] M. L. Bhaumik. Deciphering the enigma of waveparticle duality. Quanta 2016; 5(1):93-100. doi : 10.12743/quanta.v5i1.54

[35] M. L. Bhaumik. Is Schrödinger's cat alive?. Quanta 2017; 6(1):70-80. doi:10.12743/quanta.v6i1. 68.

[36] M. L. Bhaumik. How does nature accomplish spooky action at a distance?. Quanta 2018; 7(1):111117. doi:10.12743/quanta.v7i1.82.

[37] J. S. Bell. Speakable and Unspeakable in Quantum Mechanics. Cambridge University Press, Cambridge, 2004. doi : 10.1017/cbo9780511815676.

[38] J. E. Carlstrom, T. M. Crawford, L. Knox. Particle physics and the cosmic microwave background. Physics Today 2015; 68(3):28-34. doi:10.1063/ pt.3.2718.

[39] L. A. Rozema, A. Darabi, D. H. Mahler, A. Hayat, Y. Soudagar, A. M. Steinberg. Violation of heisenberg's measurement-disturbance relationship by weak measurements. Physical Review Letters 2012; 109(10):100404. doi:10.1103/PhysRevLett. 109.100404 .

[40] J. Erhart, S. Sponar, G. Sulyok, G. Badurek, M. Ozawa, Y. Hasegawa. Experimental demonstra- tion of a universally valid error-disturbance uncertainty relation in spin measurements. Nature Physics 2012; 8(3):185-189. doi:10.1038/nphys2194.

[41] M. Ozawa. Heisenberg's original derivation of the uncertainty principle and its universally valid reformulations. Current Science 2015; 109:2006-2016. arXiv: 1507.02010 doi:10.18520/v109/i11/ 2006-2016.

[42] M. Born. Statistical interpretation of quantum mechanics. Science 1955; 122(3172):675-679. doi: 10.1126/science.122.3172.675

[43] H. D. Zeh. On the interpretation of measurement in quantum theory. Foundations of Physics 1970; 1(1):69-76. doi: 10.1007/bf00708656

[44] W. H. Zurek. Decoherence, einselection, and the quantum origins of the classical. Reviews of Modern Physics 2003; 75(3):715-775. doi:10.1103/ RevModPhys.75.715.

[45] W. H. Zurek. Probabilities from entanglement, Born's rule $p_{k}=\left|\psi_{k}\right|^{2}$ from envariance. Physical Review A 2005; 71(5):052105. doi:10.1103/ PhysRevA.71.052105.

[46] M. Schlosshauer. Decoherence and the QuantumTo-Classical Transition. The Frontiers Collection. Springer, Berlin, 2007. doi:10.1007/978-3540-35775-9.

[47] M. Schlosshauer, A. Fine. On Zurek's derivation of the Born rule. Foundations of Physics 2005; 35(2):197-213. doi:10.1007/s10701004-1941-6.

[48] M. Fuwa, S. Takeda, M. Zwierz, H. M. Wiseman, A. Furusawa. Experimental proof of nonlocal wavefunction collapse for a single particle using homodyne measurements. Nature Communications 2015; 6:6665. doi : 10.1038/ncomms7665. 